\title{
Effects of particle distribution on mechanical properties of magneto-sensitive elastomers in a homogeneous magnetic field
}

\author{
D. Ivaneykd ${ }^{112}$, V. Toshcheviko $\sqrt{213}$, M. Saphiannikova ${ }^{2}, G$. Heinrich ${ }^{112}$ \\ ${ }^{1}$ Institute of Materials Science, Technical University of Dresden, 7 Helmholtz Str., 01069 Dresden, Germany \\ 2 Leibniz Institute of Polymer Research Dresden, 6 Hohe Str., 01069 Dresden, Germany \\ 3 Institute of Macromolecular Compounds, Russian Academy of Science, Bolshoi Prospect 31, V.O., \\ Saint-Petersburg, 199004, Russia
}

Received November 18, 2011, in final form March 26, 2012

\begin{abstract}
We propose a theory which describes the mechanical behaviour of magneto-sensitive elastomers (MSEs) under a uniform external magnetic field. We focus on the MSEs with isotropic spatial distribution of magnetic particles. A mechanical model is used in which magnetic particles are arranged on the sites of three regular lattices: simple cubic, body-centered cubic and hexagonal close-packed lattices. By this we extend our previous approach [Ivaneyko D. et al., Macromolecular Theory and Simulations, 2011, 20, 411] which used only a simple cubic lattice for describing the spatial distribution of the particles. The magneto-induced deformation and the Young's modulus of MSEs are calculated as functions of the strength of the external magnetic field. We show that the magneto-mechanical behaviour of MSEs is very sensitive to the spatial distribution of the magnetic particles. MSEs can demonstrate either uniaxial expansion or contraction along the magnetic field and the Young's modulus can be an increasing or decreasing function of the strength of the magnetic field depending on the spatial distribution of the magnetic particles.
\end{abstract}

Key words: magneto-sensitive elastomers, mechanical properties, modulus, modelling

PACS: $61.41 .+e, 67.30 . e r, 61.43 . B n$

\section{Introduction}

Magneto-sensitive elastomers (MSEs), also known as magnetorheological elastomers, are high-tech materials that can change their shape and mechanical behaviour under external magnetic fields [1]. Nowadays, MSEs have found a wide range of industrial applications in controllable membranes, rapidresponse interfaces designed to optimize mechanical systems and in automobile applications such as adaptive tuned vibration absorbers, stiffness tunable mounts and automobile suspensions [2-8].

Usually, MSEs consist of micron-sized magnetic particles dispersed within an elastomeric matrix. The spatial distribution of the particles inside an elastomer can be either isotropic or anisotropic (chain-like, plane-like), depending on the method of preparation [9]. MSEs with isotropic distribution of magnetic particles are synthesized by cross-linking of a polymer melt with well-dispersed magnetic particles without any external field. To obtain an MSE with chain-like distributions of particles, one should apply a strong uniaxial external magnetic field to a polymer melt before and during its cross-linking [1]. Using a complex magnetic field with a rotating vector of magnetic strength or a strong shear flow before the cross-linking procedure, one can produce an MSE with plane-like distributions of particles [10].

The magnetostriction effect (i.e., magneto-induced deformation) and the change of mechanical moduli under external magnetic field are the most significant properties of the MSEs [11]. The deformation of the MSEs can be either positive (elongation) or negative (contraction) with respect to the direction of the applied external magnetic field. Magnetostriction and the mechanical moduli of the MSEs have 
been investigated by experimental [11-16], theoretical [17-20] and simulation [21-24] studies. In theoretical studies of the mechanical behaviour of the MSEs, different analytical approaches were proposed, which can be divided into two groups: continuum-mechanics approach and microscopic approach. In the continuum-mechanics approach, electromagnetic equations are coupled with appropriate mechanical deformation equations. Thus, macroscopic homogeneity of magnetic media is assumed. This approach predicts positive magnetostriction for such complex magnetic object as MSEs. However, the continuummechanics approach is not capable of describing a local discrete spatial distribution of particles.

In the case of microscopic approach, magnetic particles are considered to be separated by a nonmagnetic matrix. Dipole-dipole interaction between the particles leads to pair-wise attraction and to repulsion of the magnetic particles depending on their mutual positions. Since dipole-dipole interactions are very sensitive to the particle positions, spatial distribution of the particles inside the matrix strongly affects the sign of magnetostriction, as it was shown in the simulation [24] and in the experiment [25]. For instance, MSEs with isotropic spatial distribution of particles demonstrate an expansion along the magnetic field, while MSEs with chain-like distribution of particles demonstrate a uniaxial contraction [1].

Recently, we have studied magnetostriction of the MSEs with isotropic and anisotropic spatial distributions of magnetic particles within the framework of a microscopic approach [26]. To describe spatial distribution of particles we have used a regular rectangular lattice model, which permits to consider "isotropic", chain-like and plane-like structures of particles. Such a regular rectangular lattice model predicts a negative magnetostriction of MSE for all distributions of particles. Our prediction for negative magnetostriction is in agreement with experimental works [13, 27, 28] for chain-like distribution. However, predictions of the rectangular lattice, which degenerates into cubic lattice for isotropic spatial distribution of particles, contradict the experimental data for magneto-induced deformation of the MSEs with an isotropic distribution of magnetic particles [20, 28]. The regular rectangular lattice is probably not capable of sufficiently describing the isotropic distribution. The reason is that any regular lattice is intrinsically anisotropic, since one always finds the lattice directions with different regular distances between the lattice sites. However, in spite of this feature, some regular lattices can possibly be used to reasonably well model the mechanical behaviour of the isotropic MSE. Therefore, in the present study we consider different lattices to describe isotropic distributions of magnetic particles in an MSE: simple cubic, body-centered cubic and hexagonal close-packed lattices. For these three different lattice models we examine magnetostriction and Young's modulus of the MSE in the presence of an external magnetic field. We construct the free energy which consists of elastic and magnetic energies. The Neo-Hooke law is used to describe entropic non-linear elasticity of polymer chains.

\section{Free energy of MSE}

\subsection{General equations}

In this section we derive an expression for the free energy of an MSE. Analysis of the free energy will permit us to study the mechanical properties of the MSE. Typical MSEs are characterized by a strong elastic coupling between the particles and the matrix. This coupling is due to the fact that magnetic particles are much larger than the mesh size of the polymer network. Since our paper is devoted to the static mechanical properties of MSEs, we consider only average displacements of particles coupled to the matrix. Under these assumptions the free energy of the deformed MSE under external magnetic field can be written in the form:

$$
F=F_{\mathrm{el}}+F_{\mathrm{m}} .
$$

The first part $F_{\mathrm{el}}$ is the elastic energy of a deformed MSE due to the entropic elasticity of polymer chains. In the present work we extend a formalism presented in reference [26] for linear deformations of MSEs to non-linear deformations. To calculate the elastic part of the free energy $F_{\mathrm{el}}$ of an MSE under finite deformation as a function of the strain we use the approach of a continuous medium. This approach means that a sample is divided into representative volumes of an elastic matrix which contain a large number of particles. Under this assumption, the free energy $F_{\mathrm{el}}$ of an MSE under non-linear deformations 
can be expressed through the Neo-Hooke law that can be written in a general form [29]:

$$
F_{\text {el }}=\frac{G_{0}}{2}\left(I_{1}-3\right),
$$

where the material parameter $G_{0}$ is the effective shear modulus of an MSE, $I_{1}$ is the first scalar invariant of the Finger strain tensor $\mathbf{B}: I_{1}=\operatorname{tr} \mathbf{B}$. The value of $G_{0}$ includes contributions of different possible effects into the elastic energy appearing under elongation of a sample: reinforcement of an elastic matrix by hard particles due to non-uniform deformation of the matrix between the particles (strain amplification), possible adhesion of a polymer matrix onto the surfaces of hard particles, etc. However, we do not discuss here how the value of $G_{0}$ depends on these effects, since this task is a special problem in the theory of elasticity for isotropic reinforced rubbers [30]. Instead we use $G_{0}$ as a phenomenological parameter of the theory assuming that it can be extracted from experimental data for elasticity of an MSE in the absence of a magnetic field. In our theory we assume an incompressible elastomeric matrix with Poisson's coefficient $v=1 / 2$. Due to the axial symmetry with respect to the external magnetic field $\overrightarrow{\mathbf{H}}$, the MSE will provide an uniaxial elongation along $\overrightarrow{\mathbf{H}}$. In this case, the first scalar invariant $I_{1}$ has the form:

$$
I_{1}=\sum_{i} \lambda_{i}^{2}
$$

where $\lambda_{x}, \lambda_{y}, \lambda_{z}$ are the elongation ratios for the deformation of an elastomer in the three principal directions.

The mechanical response of an elastomer to the magnetic field is characterized by the value of the strain $\varepsilon=\Delta l / l$, where $\Delta l$ and $l$ are the elongation and the original size, respectively, of an elastomer along the direction of the magnetic field ( $x$-axis). The condition of constant volume for elastomers [31, 32] permits us to relate the elongation ratios $\lambda_{x}, \lambda_{y}, \lambda_{z}$ as follows:

$$
\lambda_{x}=1+\varepsilon, \quad \lambda_{y}=\lambda_{z}=1 / \sqrt{1+\varepsilon} .
$$

Within the framework of Neo-Hooke approximation, the elastic energy for elastomer as a function of $\varepsilon$ can be written as:

$$
F_{\mathrm{el}}=\frac{E_{0}}{6}\left[(1+\varepsilon)^{2}+\frac{2}{1+\varepsilon}-3\right]=\frac{E_{0} \varepsilon^{2}}{2}+O\left(\varepsilon^{3}\right),
$$

where $E_{0}$ is the Young's modulus of a filled matrix: $E_{0}=3 G_{0}$ [33]. In the present theory, the value of the Young's modulus $E_{0}$ of the filled elastomer will be considered as a parameter in calculations.

The second part of the free energy in equation (2.1) $F_{\mathrm{m}}$ arises from the potential energy of magnetic particles placed in an external magnetic field. Application of a magnetic field induces an average magnetic moment in each particle. The values of the induced magnetic moments in the magnetic particles depend on the material of the particles. Magnetic particles that are usually used in preparing MSEs have micron-sizes and a multi-domain magnetic structure. Nevertheless, they are found to show very narrow hysteresis cycles which indicates a soft magnetic behaviour. In this case, the dependence of magnitude of the particle magnetization $M$ on the field strength $H$ can be described in a good approximation by the Fröhlich-Kennely equation [34,35]

$$
M=\frac{M_{\mathrm{s}}\left(\mu_{\mathrm{ini}}-1\right) H}{M_{\mathrm{s}}+\left(\mu_{\mathrm{ini}}-1\right)|H|},
$$

where $M_{\mathrm{S}}$ is the saturation magnetization and $\mu_{\text {ini }}$ is the magnetic permeability of the particles. The values of the saturation magnetization and magnetic permeability for carbonyl iron particles are well established in experiment and are equal to $M_{\mathrm{s}} \approx 1582 \mathrm{kA} / \mathrm{m}$ and $\mu_{\text {ini }} \approx 21.5$ with the average diameter of particles of $470 \pm 180 \mathrm{~nm}[36]$. Similar values were obtained for particles of the size of $2 \mu \mathrm{m}: M_{\mathrm{s}}=1990 \mathrm{kA} / \mathrm{m}$ and $\mu_{\text {ini }}=132$ [35]. Equation (2.6) can be rewritten in terms of dimensionless parameters: reduced magnetic field $h=\left(\mu_{\text {ini }}-1\right) H / M_{\mathrm{S}}$ and reduced magnetization $M / M_{\mathrm{S}}$ in the following form:

$$
\frac{M}{M_{\mathrm{s}}}=\frac{h}{1+|h|} .
$$

The dependence of $M / M_{\mathrm{S}}$ on $h$ is given in figure 1. Equation (2.7) is used below for calculations of mechanical characteristics of MSEs in an external magnetic field. 


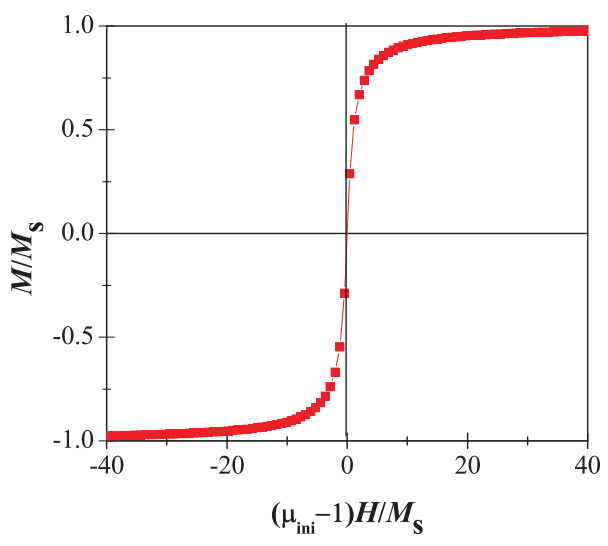

Figure 1. Reduced magnetization $M / M_{\mathrm{S}}$ as a function of a reduced magnetic field $h=\left(\mu_{\text {ini }}-1\right) H / M_{\mathrm{S}}$. Magnitude of a reduced magnetization increases with an increasing magnetic field and tends to saturation magnetization $M_{\mathrm{s}}$, when $H \rightarrow \infty$.

The interaction energy of magnetic particles in an external magnetic field consists of two contributions: the dipole-dipole interaction energy between the particles and the dipole-field interaction energy [37, 38]. However, under our assumptions given by equation [2.6), the dipole-field interaction energy $-(\overrightarrow{\mathbf{M}} \cdot \overrightarrow{\mathbf{H}})$ is independent of the strain $\varepsilon$, since the bulk magnetization of the sample $\overrightarrow{\mathbf{M}}$ is assumed to be independent of strain $\varepsilon$. Therefore, the dipole-field interaction energy does not provide a contribution to the mechanical characteristics and it can be excluded from our considerations. Thus, in our approach the magnetic energy $F_{\mathrm{m}}$ includes only dipole-dipole interaction energy per unit volume, which can be written as:

$$
F_{\mathrm{m}}=-\frac{1}{V} \frac{\mu_{\mathrm{r}} \mu_{0}}{4 \pi} \sum_{i j}\left[\frac{3\left(\overrightarrow{\mathbf{m}}_{i} \cdot \overrightarrow{\mathbf{R}}_{i j}\right)\left(\overrightarrow{\mathbf{m}}_{j} \cdot \overrightarrow{\mathbf{R}}_{i j}\right)}{\left|\overrightarrow{\mathbf{R}}_{i j}\right|^{5}}-\frac{\left(\overrightarrow{\mathbf{m}}_{i} \cdot \overrightarrow{\mathbf{m}}_{j}\right)}{\left|\overrightarrow{\mathbf{R}}_{i j}\right|^{3}}\right],
$$

where $\mu_{0}$ is permeability of the vacuum, $V$ is the volume of the sample, $\mu_{\mathrm{r}}$ is the relative permeability of the medium. In the present work we consider an elastomeric matrix to be non-magnetic. Therefore, everywhere below we set $\mu_{\mathrm{r}}=1$. Here $\overrightarrow{\mathbf{m}}_{i}$ and $\overrightarrow{\mathbf{m}}_{j}$ are dipole moments of $i$-th and $j$-th magnetic particles, $\overrightarrow{\mathbf{R}}_{i j}$ is the radius vector that joins the $i$-th and $j$-th magnetic particles. The radius vectors $\overrightarrow{\mathbf{R}}_{i j}$ depend on the macroscopic deformation $\varepsilon$. To relate $\overrightarrow{\mathbf{R}}_{i j}$ with $\varepsilon$ we use an approximation of affinity of deformation [31, 32], as well as incompressibility of elastomers:

$$
\begin{aligned}
& \left(R_{i j}\right)_{x}=\left(R_{i j}^{0}\right)_{x} \lambda_{x}=\left(R_{i j}^{0}\right)_{x}(1+\varepsilon), \\
& \left(R_{i j}\right)_{y}=\left(R_{i j}^{0}\right)_{y} \lambda_{y}=\left(R_{i j}^{0}\right)_{y}(1+\varepsilon)^{-\frac{1}{2}} \\
& \left(R_{i j}\right)_{z}=\left(R_{i j}^{0}\right)_{z} \lambda_{z}=\left(R_{i j}^{0}\right)_{z}(1+\varepsilon)^{-\frac{1}{2}}
\end{aligned}
$$

where $\left(R_{i j}\right)_{\xi}$ and $\left(R_{i j}^{0}\right)_{\xi}$ are the components of vectors that join two magnetic particles after and before deformation, respectively, $(\xi=x, y, z)$. Summation in equation 2.8 is performed over all pairs of particles. To simplify the calculations, we consider regular spatial distributions of particles. In particular, we use three different lattices in order to study the effects of particle distribution on the magneto-mechanical properties of the MSEs in a homogeneous magnetic field.

\section{2. "Isotropic" distributions of magnetic particles: different lattice models}

In contrast to our previous study [26], which used only a cubic lattice to describe the isotropic distribution of magnetic particles inside the MSE, we consider here different lattice models (see figure 2). In these models, it is assumed that the magnetic particles in an isotropic non-deformed MSE are located at the sites of simple cubic (SC), body-centered cubic (BCC) and hexagonal close-packed (HCP) lattices. Let $a$ be the edge length in the three lattices [figure2(b)]. 


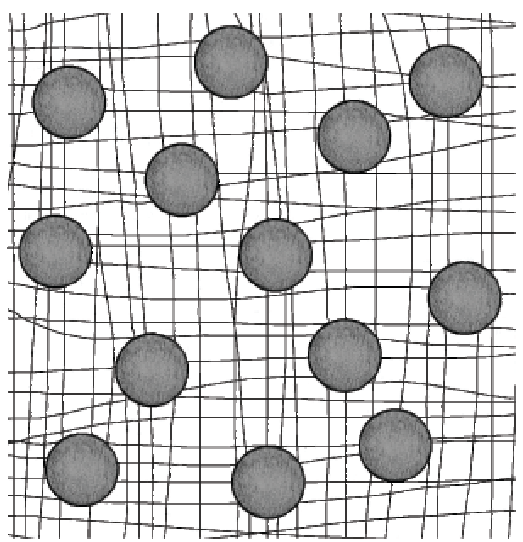

(a)

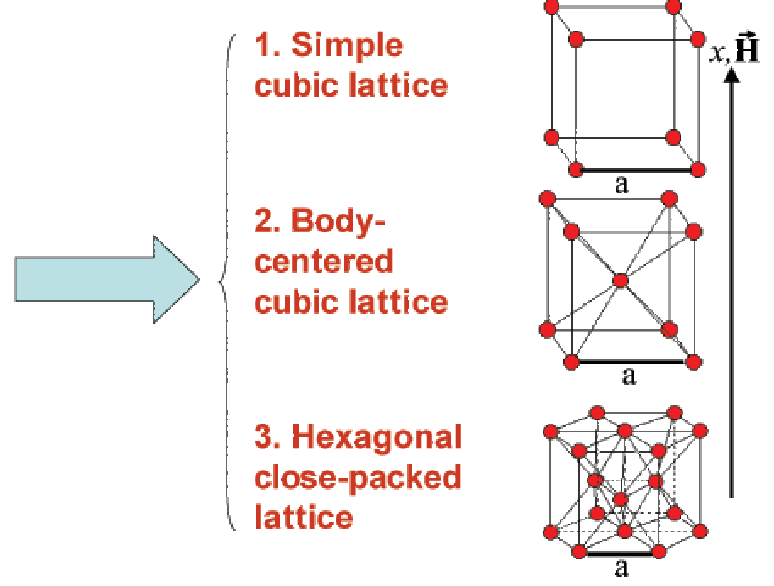

(b)

Figure 2. Schematic illustration of an MSE with isotropic spatial distribution of particles (a) and the lattice models (simple cubic, body-centered cubic and hexagonal lattices) for modelling the mechanical properties of an MSE (b).

For simplicity we assume that all particles are the same and have a spherical form; $r$ is the radius of particles. The value of $r$ characterizes the average size of particles in a real elastomer. Then, the volume fraction, $\phi$, of the particles distributed in the matrix is given by:

$$
\phi=\gamma \frac{v_{0}}{a^{3}},
$$

where $v_{0}=\frac{4}{3} \pi r^{3}$ is the volume of a particle. The factor $\gamma$ depends on the type of the lattice:

$$
\gamma_{\mathrm{SC}}=1, \quad \gamma_{\mathrm{BCC}}=2, \quad \gamma_{\mathrm{HCP}}=2 \sqrt{2}
$$

Due to the symmetries of the infinite lattices to be used (translational symmetry and the axial symmetry around the vector $\overrightarrow{\mathbf{H}}$ ), the induced magnetic dipoles $\overrightarrow{\mathbf{m}}_{i}$ and $\overrightarrow{\mathbf{m}}_{j}$ are directed along the external field $\overrightarrow{\mathbf{H}}$ [ $x$-axis in figure 2(b)] and their absolute values are identical $m_{i}=m_{j}=v_{0} M$, with $M$ being the magnetization of each particle. Besides, a regular arrangement of the magnetic particles on the sites of the lattices makes it possible to simplify the summation in the equation (2.8). To perform summation over indexes $i$ and $j$ we choose $j$-th particle and make summation over index $i$. Due to the translational symmetry for infinite lattices, the contribution to the magnetic energy of the dipole-dipole interaction of a given $j$-th particle with other particles around it does not depend on the number $j$. Thus, the double sum over pairs of indexes $i$ and $j$ is reduced to a simple sum over index $i$ multiplied by $N$, where $N$ is the number of all particles. Then, equation (2.8) can be rewritten in the form:

$$
F_{\mathrm{m}}=-u_{0} v_{0}^{2} c\left(\frac{M}{M_{\mathrm{S}}}\right)^{2} \sum_{i}\left[\frac{3\left(\overrightarrow{\mathbf{R}}_{i}\right)_{x}^{2}-\left|\overrightarrow{\mathbf{R}}_{i}\right|^{2}}{\left|\overrightarrow{\mathbf{R}}_{i}\right|^{5}}\right],
$$

where $c=N / V$ is the concentration of particles. Here, for convenience we calculate the radius vectors from the origin of a lattice and simplify the notation as $\overrightarrow{\mathbf{R}}_{i j} \equiv \overrightarrow{\mathbf{R}}_{i}$. We introduce the parameter $u_{0}$ :

$$
u_{0}=\frac{\mu_{0} M_{\mathrm{s}}^{2}}{4 \pi}
$$

that defines the characteristic density of energy of magnetic interaction. For $M_{\mathrm{S}} \approx 2 \times 10^{6} \mathrm{~A} / \mathrm{m}$ we obtain $u_{0}=4 \times 10^{5} \mathrm{~Pa}$. Below, we will show that mechanical behaviour of an MSE in the magnetic field is determined by a dimensionless parameter $E_{0} / u_{0}$, i.e., by the ratio between characteristic values of the elastic and magnetic energies. 
The radius vector $\overrightarrow{\mathbf{R}}_{i}$ in equation (2.14) is related with the radius vector $\overrightarrow{\mathbf{R}}_{i}^{0}$ in a non-deformed MSE by equations (2.9)-2.11). For simple cubic, body-centered cubic and hexagonal close-packed lattices, the value $\overrightarrow{\mathbf{R}}_{i}^{0}$ can be presented in the form:

$$
\overrightarrow{\mathbf{R}}_{i}^{0}=a \cdot \overrightarrow{\mathbf{r}}_{i}
$$

The dimensionless vector $\overrightarrow{\mathbf{r}}_{i}$ runs now over all sites of the infinite lattice with the edge length $a=1$ except $\overrightarrow{\mathbf{r}}_{i}=0$. Using the Fröhlich-Kennely equation (2.6) for reduced magnetization $M / M_{\mathrm{s}}$, we express the magnetic energy $F_{\mathrm{m}}$ as a function of a reduced magnetic field $h$ and the strain $\varepsilon$ :

$$
F_{\mathrm{m}}=u_{0} \phi^{2}\left(\frac{h}{1+|h|}\right)^{2} f(\varepsilon)
$$

where the dimensionless function $f(\varepsilon)$ has the following form:

$$
f(\varepsilon)=-\frac{1}{\gamma}(1+\varepsilon)^{\frac{3}{2}} \sum_{r_{i} \neq 0} \frac{2(1+\varepsilon)^{3}\left(r_{i}\right)_{x}^{2}-\left(r_{i}\right)_{y}^{2}-\left(r_{i}\right)_{z}^{2}}{\left[(1+\varepsilon)^{3}\left(r_{i}\right)_{x}^{2}+\left(r_{i}\right)_{y}^{2}+\left(r_{i}\right)_{z}^{2}\right]^{\frac{5}{2}}} .
$$

We recall that the vector $\overrightarrow{\mathbf{r}}_{i}$ runs over the sites of different lattices with $a=1$. The value of function $f(\varepsilon)$ depends on the type of the lattice, since the components of the vectors $\overrightarrow{\mathbf{r}}_{i}$ depend on the lattice. The vector $\overrightarrow{\mathbf{r}}_{i}$ for three lattices can be expressed in the following form:

$$
\overrightarrow{\mathbf{r}}_{i}=\vec{a} \cdot i_{a}+\vec{b} \cdot i_{b}+\vec{c} \cdot i_{c}
$$

where $i_{a}, i_{b}$ and $i_{c}$ are the integer numbers, $\vec{a}, \vec{b}$ and $\vec{c}$ are the basis vectors. The basis vectors are presented in figure 3 and have their coordinates:

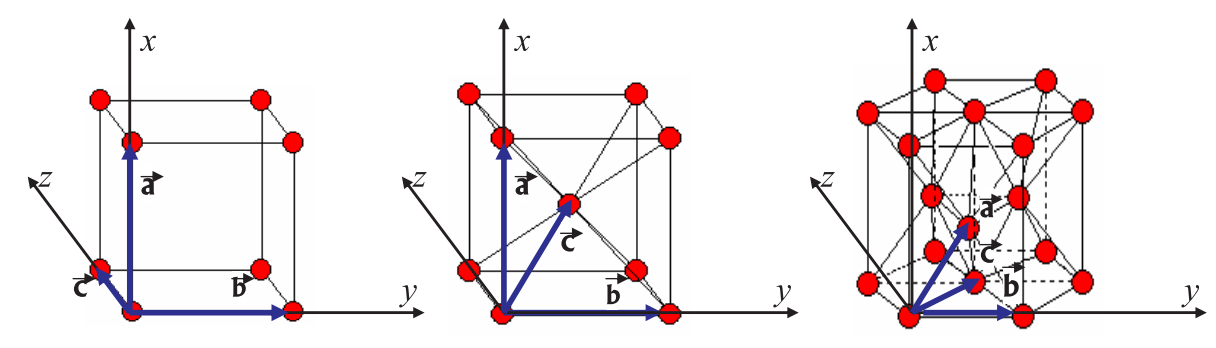

Figure 3. (Color online) Basis vectors $\vec{a}, \vec{b}$ and $\vec{c}$ introduced for the simple cubic, body-centered cubic and hexagonal close-packed lattices with the unit edge length, $|\vec{b}|=1$.

- Simple cubic lattice

$$
\vec{a}=(1,0,0), \quad \vec{b}=(0,1,0), \quad \vec{c}=(0,0,1)
$$

- Body-centered cubic lattice

$$
\vec{a}=(1,0,0), \quad \vec{b}=(0,1,0), \quad \vec{c}=\left(\frac{1}{2}, \frac{1}{2}, \frac{1}{2}\right) ;
$$

- Hexagonal close-packed lattice

$$
\vec{a}=\left(\sqrt{\frac{2}{3}}, \frac{1}{2}, \frac{1}{2 \sqrt{3}}\right), \quad \vec{b}=(0,1,0), \quad \vec{c}=\left(0, \frac{1}{2}, \frac{\sqrt{3}}{2}\right) .
$$

For different lattices, the function $f(\varepsilon)$ was analyzed numerically. Below we consider the magnetostriction and the Young's modulus of the MSE in the presence of an external magnetic field depending on the type of the lattice model. 


\subsection{Free energy as a function of deformation}

Using equation (2.5) for elastic energy and equation (2.17) for magnetic energy, we obtain free energy of the MSE in the following form:

$$
F=\frac{E_{0}}{6}\left[(1+\varepsilon)^{2}+\frac{2}{1+\varepsilon}-3\right]+u_{0} \phi^{2}\left(\frac{h}{1+|h|}\right)^{2} f(\varepsilon),
$$

where the values of the function $f(\varepsilon)$ vary for the simple cubic, body-centered cubic and hexagonal close-packed lattices. We calculate the reduced free energy, $F / u_{0}$, of an isotropic MSE as a function of the strain $\varepsilon$ for the three types of initial lattices at fixed values $\phi=0.05$ and $E_{0} / u_{0}=2.5$ and at different values of the reduced magnetic field $h=0,0.25,0.70,1.5,4,40$ that correspond to the values $M / M_{\mathrm{S}}=0,0.2,0.4,0.6,0.8,1.0$, respectively. One can see in figure 4 that application of the magnetic field leads to the shift of the minimum of free energy. The minimum of free energy determines the equilib-

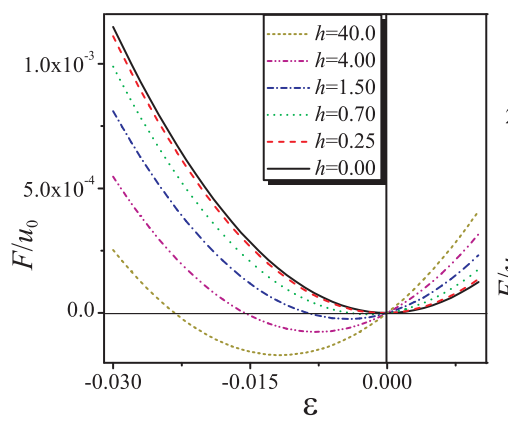

(a)

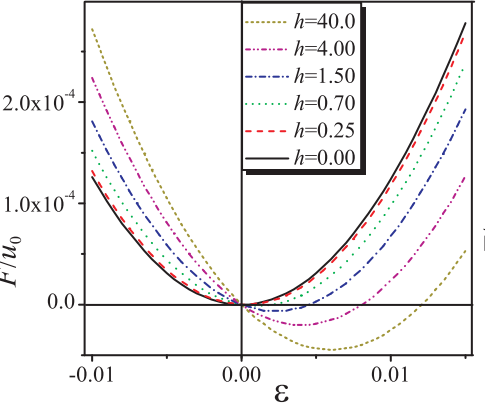

(b)

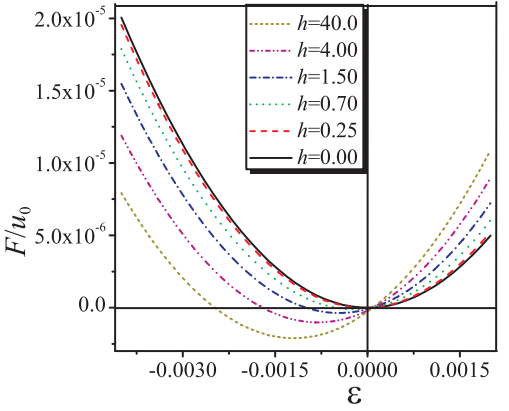

(c)

Figure 4. (Color online) Reduced free energy, $F / u_{0}$, of an isotropic MSE as a function of the strain $\varepsilon$ calculated for three types of initial lattices: simple cubic lattice (a), body-centered cubic lattice (b) and hexagonal lattice (c) at different values of the reduced magnetic field $h=\left(\mu_{\mathrm{ini}}-1\right) H / M_{\mathrm{S}}$.

rium elongation of an MSE, $\varepsilon_{\text {eq }}$. One can see that the value and the sign of the $\varepsilon_{\text {eq }}$ depend on the lattice: $\varepsilon_{\text {eq }}<0$ for the simple cubic and hexagonal close-packed lattices and $\varepsilon_{\text {eq }}>0$ for the body-centered cubic lattice. This means that both simple cubic and hexagonal close-packed lattices predict a contraction of an isotropic MSE along the magnetic field $\overrightarrow{\mathbf{H}}\left(\varepsilon_{\text {eq }}<0\right)$, whereas the body-centered cubic lattice predicts an expansion of an isotropic MSE along the magnetic field $\overrightarrow{\mathbf{H}}\left(\varepsilon_{\text {eq }}>0\right)$.

The analysis of $\varepsilon_{\text {eq }}$ as a function of the reduced magnetic field $h$, the volume fraction $\phi$ and parameter $E_{0} / u_{0}$ for different lattices are given below.

\section{Mechanical behaviour of MSEs in a homogeneous magnetic field}

\subsection{Magnetostriction of the MSE}

In this section we study the magnetostriction effect in the MSE with isotropic spatial distribution of magnetic particles. We calculate the equilibrium elongation of the MSE from the minimum of free energy $F$ :

$$
\left.\frac{\partial F}{\partial \varepsilon}\right|_{\varepsilon=\varepsilon_{\mathrm{eq}}}=0
$$




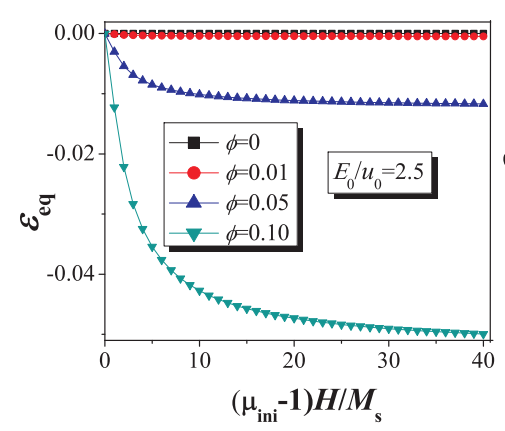

(a)

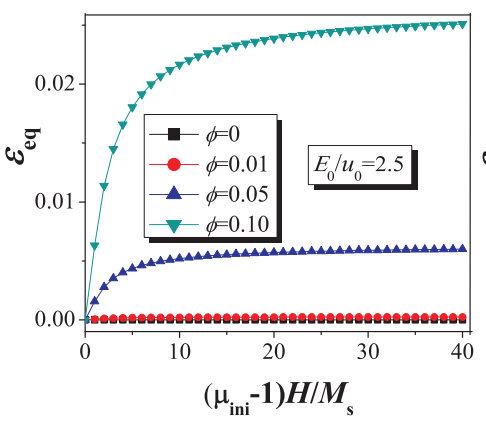

(b)

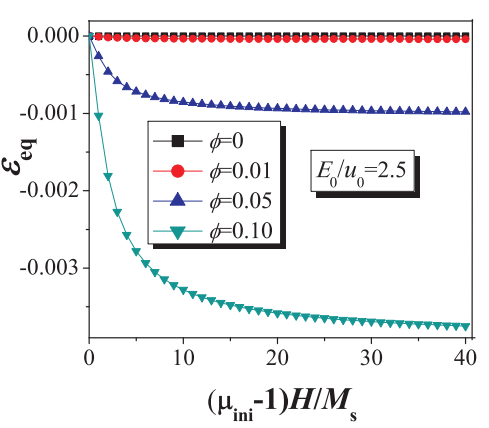

(c)

Figure 5. (Color online) Dependence of the equilibrium elongation $\varepsilon_{\text {eq }}$ on the reduced magnetic field $h=\left(\mu_{\mathrm{ini}}-1\right) H / M_{\mathrm{S}}$ at different volume fractions $\phi$, calculated for three types of initial lattices: simple cubic lattice (a), body-centered cubic lattice (b) and hexagonal close-packed lattice (c).

Using equations (2.23) and (2.18), the equation (3.1) for the equilibrium elongation $\varepsilon_{\text {eq }}$ can be rewritten as follows:

$$
\begin{aligned}
& \frac{E_{0}}{3}\left[1+\varepsilon_{\mathrm{eq}}-\frac{1}{\left(1+\varepsilon_{\mathrm{eq}}\right)^{2}}\right]+u_{0} \phi^{2}\left(\frac{h}{1+|h|}\right)^{2} \frac{1}{\gamma} \sqrt{1+\varepsilon_{\mathrm{eq}}} \\
& \quad \times \sum_{r_{i} \neq 0} \frac{12\left(1+\varepsilon_{\mathrm{eq}}\right)^{6}\left(r_{i}\right)_{x}^{4}-30\left(r_{i}\right)_{x}^{2}\left(1+\varepsilon_{\mathrm{eq}}\right)^{3}\left[\left(r_{i}\right)_{y}^{2}+\left(r_{i}\right)_{z}^{2}\right]+3\left[\left(r_{i}\right)_{y}^{2}+\left(r_{i}\right)_{z}^{2}\right]^{2}}{2\left[\left(1+\varepsilon_{\mathrm{eq}}\right)^{3}\left(r_{i}\right)_{x}^{2}+\left(r_{i}\right)_{y}^{2}+\left(r_{i}\right)_{z}^{2}\right]^{\frac{7}{2}}}=0 .
\end{aligned}
$$

Dividing both the left- and right-hand sides of equation (3.2) by the factor $u_{0}$, one can see that the equilibrium elongation $\varepsilon_{\text {eq }}$ depends on the elastic modulus $E_{0}$ and on the magnetic parameter $u_{0}$ through their dimensionless ratio $E_{0} / u_{0}$. The last equation we solve numerically at varied values of the reduced magnetic field $h$. Figure 5 shows dependences of the equilibrium elongation $\varepsilon_{\mathrm{eq}}$ on the reduced magnetic field $h$ at the values of parameter $E_{0} / u_{0}=2.5$ and volume fraction $\phi=0,0.01,0.05$ and 0.1 , calculated for the three types of lattice models. Figure 6 shows dependences of the equilibrium elongation $\varepsilon_{\text {eq }}$ on the reduced magnetic field $h$ at the values of volume fraction $\phi=0.05$ and parameters $E_{0} / u_{0}=1.0,2.5,5$ and 10, calculated for the three types of lattice models. One can see that for simple cubic and hexagonal close-packed lattices, an MSE is uniaxially contracted along the direction of the external magnetic field, $\varepsilon_{\text {eq }}<0$ [figure 5](a), (c) and 6(a), (c)], while for the body-centered cubic lattice, an MSE uniaxially expands along the direction of the external magnetic field, $\varepsilon_{\text {eq }}>0$ [figure $5(\mathrm{~b})$ and 6 (b)].

Different signs of magnetostriction of MSEs with different spatial "isotropic" distributions of particles (simple cubic, body-centered cubic and hexagonal close-packed lattices) can be explained by the fact that the mechanical behaviour of MSEs is determined mainly by the mutual attraction and repulsion of the

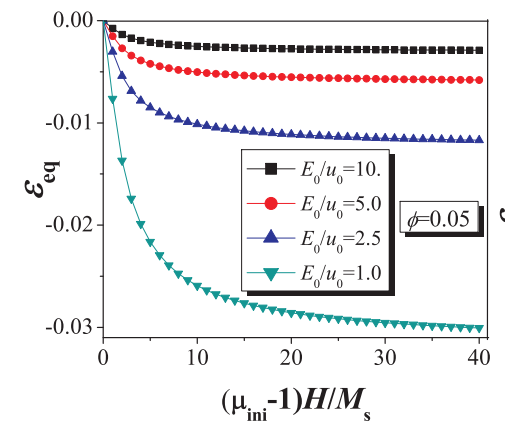

(a)

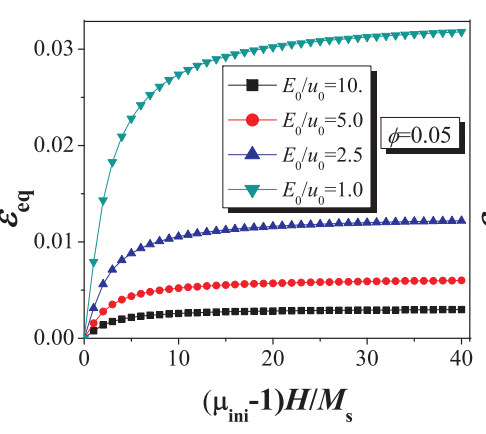

(b)

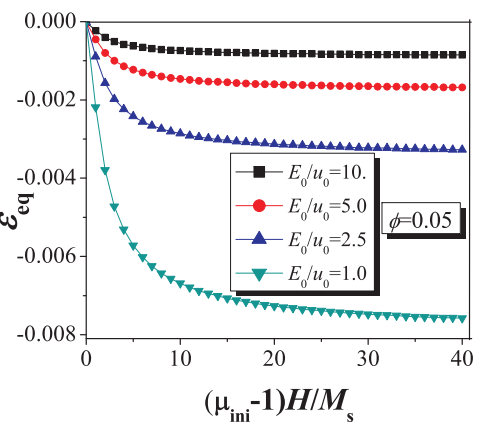

(c)

Figure 6. (Color online) Same as figure 5 but at different values of the parameter $E_{0} / u_{0}$ and at fixed volume fraction $\phi=0.05$. 
nearest particles as it is illustrated in figure 7 In a simple cubic lattice, the nearest particles to a given one (B) are the particles A and C as presented in figure7 7 Such configurations lead to the contraction of an MSE along the magnetic field which is in accord with our results presented in figures[5(a) and 6 (a). In the body-centered cubic and hexagonal close-packed lattices there exist some extra particles whose positions are determined by the angle $\theta$ (see figure 7). Depending on the value of $\theta$, either contraction or elongation of an MSE is possible. One can show that interaction between the particles B and D results in the expansion at $32^{\circ}<\theta<72^{\circ}$ and in contraction at $0<\theta<32^{\circ}, 72^{\circ}<\theta<90^{\circ}$. Therefore, the body-centered cubic lattice predicts an expansion since $\theta_{\mathrm{BCC}}=54.7^{\circ}$. For a hexagonal close-packed lattice, the nearest particles

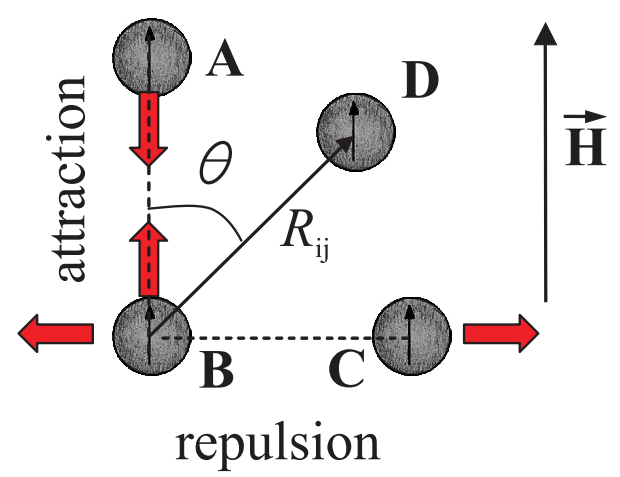

Figure 7. Attraction and repulsion of magnetic particles in an MSE depending on their mutual positions. which lie at the angles $\theta_{\mathrm{HCP}}=35^{\circ}$ provide an expansion and the nearest particles which lie at $\theta_{\mathrm{HCP}}=90^{\circ}$ provide a contraction. We have shown that the interplay between two different contributions of the nearest particles leads to the contraction. Thus, the sign of magnetostriction strongly depends on the local spatial distribution of magnetic particles.

Furthermore, one can see in figure 5] that the increase of the volume fraction $\phi$ results in an increase of the equilibrium elongation $\left|\varepsilon_{\text {eq }}\right|$, when $h$ is fixed. Figure 6 shows that the increase of the parameter $E_{0} / u_{0}$ results in a decrease of matrix deformation $\left|\varepsilon_{\text {eq }}\right|$, when $h$ is fixed. These results are explained by the fact that the relative contribution of magnetic interaction becomes larger at higher values of $\phi$ and at lower values of $E_{0} / u_{0}$.

\subsection{Young's modulus of the MSE}

In the case of a tensile deformation, we consider such geometry when the mechanical force is applied along the external magnetic field $\overrightarrow{\mathbf{H}}$, i.e., along the $x$-axis in our case (see figure 2). The Young's modulus $E$ can be obtained as the second derivative of free energy with respect to $\varepsilon$, where $\varepsilon=\varepsilon_{\text {eq }}+\delta \varepsilon$ :

$$
E=\left.\frac{\partial^{2} F}{\partial \varepsilon^{2}}\right|_{\varepsilon=\varepsilon_{\mathrm{eq}}},
$$

which yields

$$
\begin{aligned}
E= & \frac{E_{0}}{3}\left[1+\frac{2}{\left(1+\varepsilon_{\mathrm{eq}}\right)^{3}}\right]-u_{0} \phi^{2}\left(\frac{h}{1+|h|}\right)^{2} \frac{3}{4 \sqrt{1+\varepsilon_{\mathrm{eq}}}} \sum_{r_{i} \neq 0}\left\{\left[32\left(r_{i}\right)_{x}^{6}\left(1+\varepsilon_{\mathrm{eq}}\right)^{9}-\left[\left(r_{i}\right)_{y}^{2}+\left(r_{i}\right)_{z}^{2}\right]^{3}\right.\right. \\
& \left.\left.-192\left(r_{i}\right)_{x}^{4}\left[\left(r_{i}\right)_{y}^{2}+\left(r_{i}\right)_{z}^{2}\right]\left(1+\varepsilon_{\mathrm{eq}}\right)^{6}+90\left(r_{i}\right)_{x}^{2}\left[\left(r_{i}\right)_{y}^{2}+\left(r_{i}\right)_{z}^{2}\right]^{2}\left(1+\varepsilon_{\mathrm{eq}}\right)^{3}\right]\left[\left(r_{i}\right)_{x}^{2}\left(1+\varepsilon_{\mathrm{eq}}\right)^{3}+\left(r_{i}\right)_{y}^{2}+\left(r_{i}\right)_{z}^{2}\right]^{-\frac{9}{2}}\right\} .
\end{aligned}
$$

One can see that the ratio $E / E_{0}$ depends on the parameters $E_{0}$ and $u_{0}$ through their dimensionless ratio $E_{0} / u_{0}$.

We have numerically calculated $E$ as a function of the reduced magnetic field $h$ using equation (3.4). Figure 8 shows the dependence of the Young's modulus $E$ on the reduced magnetic field $h$ at the values of parameter $E_{0} / u_{0}=2.5$ and volume fraction $\phi=0,0.01,0.05$ and 0.1 , calculated for three types of initial lattices: simple cubic, body-centered cubic and hexagonal close-packed lattices. Figure 9 is the same as figure 8 but at a fixed value of the volume fraction $\phi=0.05$ and at varied values of $E_{0} / u_{0}=1.0,2.5,5$ and 10. One can see that the Young's modulus $E$ decreases for the simple cubic [figure 8(a) and 9 (a)] and the body-centered cubic [figure 8(b) and 9](b)] lattices and increases for the hexagonal close-packed lattice [figure 8(c) and 9 (c)]. Different behaviour of $E$ as a function of a reduced magnetic field $h$ for the three lattices is caused by complex combinations of interactions between particles of different mutual positions (see figure7). 


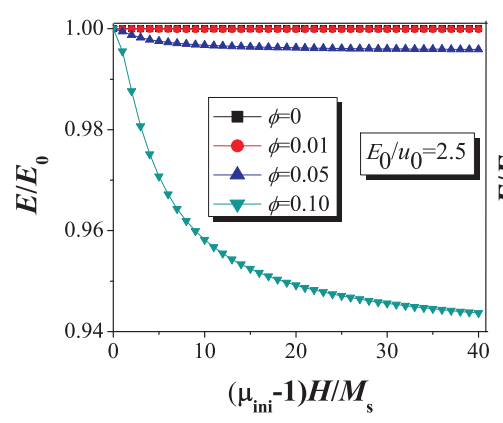

(a)

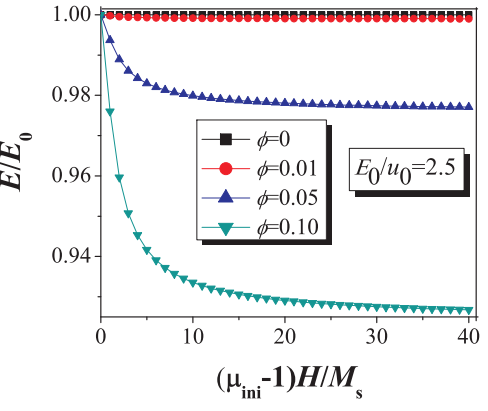

(b)

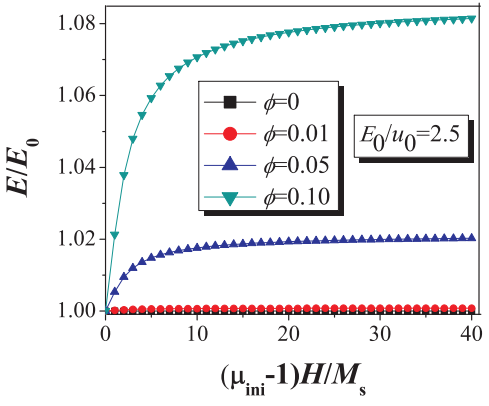

(c)

Figure 8. (Color online) Dependence of the Young's modulus $E$ on the reduced magnetic field $h=\left(\mu_{\mathrm{ini}}-\right.$ 1) $H / M_{\mathrm{S}}$ at different volume fractions $\phi$, calculated for three types of initial lattices: simple cubic lattice (a), body-centered cubic lattice (b) and hexagonal lattice (c).

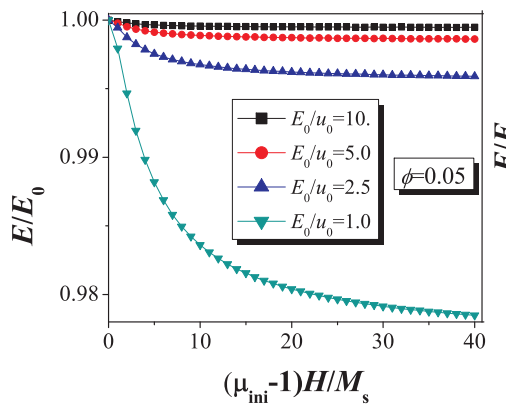

(a)

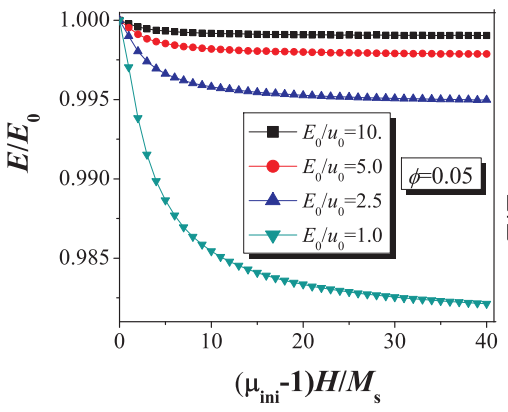

(b)

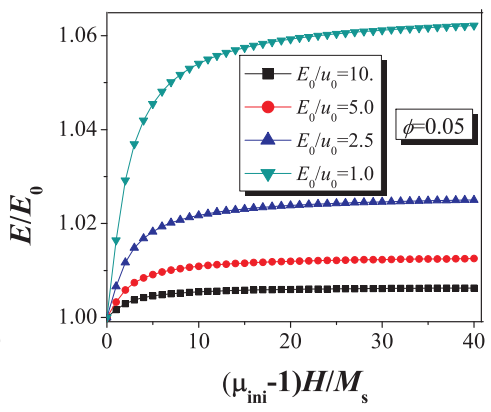

(c)

Figure 9. (Color online) Same as figure 8 but at different values of the parameter $E_{0} / u_{0}$ and at fixed volume fraction $\phi=0.05$.

Furthermore, it can be seen in figure 8 that an increase of the volume fraction $\phi$ leads to an increase of absolute values of the change of the modulus $\left|E-E_{0}\right|$ for all distributions at fixed $h$. Figure 9 shows that an increase of the parameter $E_{0} / u_{0}$ results in a decrease of absolute values of the change of the modulus $\left|E-E_{0}\right|$ for all distributions at fixed $h$. These results are explained by the fact that the relative contribution of the magnetic energy to the modulus increases at increasing values of the volume fraction $\phi$ and decreases at increasing values of the parameter $E_{0} / u_{0}$.

\section{Conclusion}

In this paper we have studied the mechanical properties of magneto-sensitive elastomers with isotropic distribution of the magnetic particles in an external magnetic field. We have used a model in which magnetic particles are located at the sites of regular lattices. Different types of the lattice models have been considered: simple cubic, body-centered cubic and hexagonal close-packed lattices. Such lattice distributions of particles can be prepared in experiment as was shown in [9]. We show that magneto-induced deformation and the Young's modulus of the MSE strongly depend on spatial distribution of magnetic particles. A simple cubic lattice model predicts a contraction of the MSE with isotropic distribution of magnetic particles along the direction of a homogeneous magnetic field. It predicts that the Young's modulus decreases with an increase of the magnetic field; the same result is obtained for the body-centered cubic model. However, in contrast to the simple cubic lattice, the body-centered cubic lattice model provides an expansion of the MSE along the direction of a magnetic filed. The hexagonal lattice model shows that MSEs with isotropic particle distribution contract along the external magnetic field, while its Young's 
modulus increases. These findings may explain different signs of magnetostriction observed in experiments with differently prepared MSEs [25].

\section{Acknowledgements}

This work was supported by funds of European Union and the Free State of Saxony (SAB project ECEMP B4 no. 13854/2379).

\section{References}

1. Filipcsei G., Csetneki I., Szilágyi A., Zrínyi M., Adv. Polym. Sci., 2007, 206, 137; doi10.1007/12_2006_104

2. Carlson D.J., Jolly M.R., Mechatronics, 2000, 10, 555; doi 10.1016/S0957-4158(99)00064-1

3. Ruddy C., Ahearne E., Byrne G. - In: The 24th International Manufacturing Conference, Waterford, 2007.

4. Deng H.X., Gong X.L., J. Intell. Mater. Syst. Struct., 2007, 18, 1205; doi 10.1177/1045389X07083128

5. Chen L., Gong X., J. Cent. South Univ. Technol., 2008, 15, 271; doi 10.1007/s11771-008-0361-8

6. Ni Z.C., Gong X.L., Li J.F., Chen L., J. Intell. Mater. Syst. Struct., 2009, 20, 1195; doi 10.1177/1045389X09104790

7. Dong X.M., Yu M., Liao C.R., Chen W.M., Trans. Nonferrous Met. Soc. China, 2009, 19, s611; doi 10.1016/S1003-6326(10)60118-5

8. Xu Z., Gong X., Liao G., Chen X., J. Intell. Mater. Syst. Struct., 2010, 21, 1039; doi 10.1177/1045389X10375485

9. Zhang X., Peng S., Wen W., Li W., Smart Mater. Struct., 2008, 17, 045001; doi 10.1088/0964-1726/17/4/045001

10. Kulichikhin V.G., Semakov A.V., Karbushev V.V., Platé N.A., Picken S.J., Polym. Sci. Ser. A Polym. Phys., 2009, 51, No. 11-12, 1303; doi 10.1134/S0965545X09110169

11. Guan X., Dong X., Ou J., J. Magn. Magn. Mater., 2008, 320, 158; doi 10.1016/j.jmmm.2007.05.043

12. Bednarek S., Appl. Phys. A, 1999, 68, 63; doi 10.1007/s003390050854

13. Coquelle E., Bossis G., J. Adv. Sci., 2005, 17, No 1-2, 132; doi 10.2978/jsas.17.132

14. Martin J.E., Anderson R.A., Read D., Gulley G., Phys. Rev. E, 2006, 74, 051507; doi 10.1103/PhysRevE.74.051507

15. Abramchuk S., Kramarenko E., Stepanov G., Nikitin L.V., Filipcsei G., Khokhlov A.R., Zrínyi M., Polym. Advan. Technol., 2007, 18, 883; doi 10.1002/pat.924.

16. Stepanov G.V., Abramchuk S.S., Grishin D.A., Nikitin L.V., Kramarenko E.Y., Khokhlov A.R., Polymer, 2007, 48, 488; doi 10.1016/j.polymer.2006.11.044

17. Raikher Y.L., Stolbov O.V., Tech. Phys. Lett., 2000, 26, 156; doi 10.1134/1.1262774

18. Borcea L., Bruno O., J. Mech. Phys. Solids, 2001, 49, 2877; doi 10.1016/S0022-5096(01)00108-9

19. Kankanala S.V., Triantafyllidis N., J. Mech. Phys. Solids, 2004, 52, 2869; doi 10.1016/j.jmps.2004.04.007

20. Diguet G., Beaugnon E., Cavaillé J.Y., J. Magn. Magn. Mater., 2010, 322, 3337; doi 10.1016/j.jmmm.2010.06.020

21. Coquelle E., Bossis G., Szabo D., Giulieri F., J. Mater. Sci., 2006, 41, 5941; doi 10.1007/s10853-006-0329-8

22. Stepanov G.V., Borin D.Y., Raikher Y.L., Melenev P.V., Perov N.S., J. Phys. Condens. Matter, 2008, 20, 204121; doi 10.1088/0953-8984/20/20/204121

23. Raikher Y.L., Stolbov O.V., J. Phys. Condens. Matter, 2008, 20, 204126; doi 10.1088/0953-8984/20/20/204126

24. Raikher Y.L., Stolbov O.V., Vychyslitelnaja Mekhanika Sploshnykh Sred, 2009, 2, No. 2, 85 (in Russian).

25. Safronov A.P., Terziyan T.V., Istomina A.S., Beketov I.V., Polym. Sci. Ser. A Polym. Phys., 2012, 54, 26; doi 10.1134/S0965545X12010063.

26. Ivaneyko D., Toshchevikov V.P., Saphiannikova M., Heinrich G., Macromol. Theory Simul., 2011, 20, 411; doi 10.1002/mats.201100018

27. Jolly M.R., Carlson J.D., Muñoz B.C., Bullions T.A., J. Intell. Mater. Syst. Struct., 1996, 7, 613; doi $10.1177 / 1045389 X 9600700601$

28. Zhou G.Y., Jiang Z.Y., Smart Mater. Struct., 2004, 13, 309; doi 10.1088/0964-1726/13/2/009

29. Szabó D., Zrínyi M., Int. J. Mod. Phys. B, 2002, 16, No. 16 \& 17, 2616; doi 10.1142/S0217979202012748

30. Vilgis T.A., Heinrich G., Klüppel M., Reinforcement of Polymer Nano-composites: Theory, Experiments and Applications. Cambridge University Press, Cambridge-New York, 2009.

31. Treloar L.R.G., The Physics of Rubber Elasticity, 2nd edn. Clarendon Press, Oxford, 1958.

32. Doi M., Edwards S.F., The Theory of Polymer Dynamic. Clarendon Press, Oxford, 1986.

33. Varga Z., Filipcsei G., Zrínyi M., Polymer, 2006, 47, 227; doi 10.1016/j.polymer.2005.10.139

34. Jiles D., Introduction to Magnetism and Magnetic Materials. Chapman and Hall Publishers, London and New York. 1998.

35. Bossis G., Abbo C., Cutillas S., Lacis S., Métayer C. - In: Proc. of the 7th International Conference on Electro- 
Rheological Fluids and Magneto-Rheological Suspensions: Honolulu, Hawaii, July 19-23, 1999, World Scientific Publishing, Singapore, 1999.

36. Arias J.L., Gallardo V., Linares-Molinero F., Delgado A.V., J. Colloid Interface Sci., 2006, 299, 599; doi 10.1016/j.jcis.2006.03.005.

37. Jackson J.D., Classical Electrodynamics, 3rd edn. John Wiley \& Sons, New York, 1998.

38. Froltsov V.A., Blaak R., Likos C.N., Löwen H., Phys. Rev. E, 2003, 68, 061406; doi 10.1103/PhysRevE.68.061406

\title{
Вплив розподілу частинок на механічні властивості магнітночутливих еластомерів в однорідному магнітному полі
}

\author{
Д. Іванейко ${ }^{122}$, В.П. Тощевіков 213, М. Саф'янніков $d^{21}$, г. Гайнріх $x^{112}$ \\ ${ }^{1}$ Інститут Матеріалознавства, Дрезденський Технічний Університет, \\ вул. Гельмгольца, 7, 01069 Дрезден, Німеччина \\ 2 Дрезденський Інститут полімерних досліджень ім. Ляйбніца, вул. Гоге, 6, 01069 Дрезден, Німеччина \\ 3 Інститут високомолекулярних сполук, Російська Академія Наук, \\ Большой пр., 31, 199004 Санкт-Петербург, Росія
}

\begin{abstract}
Ми пропонуємо теорію, яка описує механічну поведінку магнітночутливих еластомерів (МЧЕ) з ізотропним розподілом частинок в однорідному магнітному полі. Використовуються три механічні моделі, в яких магнітні частинки поміщено у вузлах відповідно трьох періодичних ґраток: простої кубічної, об'ємоцентрованої кубічної та щільно впакованої гексаґональної. Цим ми розвиваємо наш попередній підхід [Ivaneyko D. et al., Macromolecular Theory and Simulations, 2011, 20, 411], в якому ми використали лише просту кубічну ґратку для опису просторового розподілу частинок. Деформація, яка виникає під впливом магнітного поля, та модуль Юнга в МЧЕ розраховані для різних напруженостей магнітного поля. Ми показали, що магнітномеханічна поведінка МЧЕ сильно залежить від просторового розміщення магнітних частинок. МЧЕ демонструє одновісне видовження або стиск вздовж магнітного поля, а модуль Юнга зростає або спадає при збільшенні напруженості магнітного поля залежно від просторового розподілу магнітних частинок.
\end{abstract}

Ключові слова: магнітночутливі еластомери, механічні властивості, модуль, моделювання 